\title{
Regioselective combination of 1,3-dinucleophiles such as 2- mercaptobenzimidazole, 5-mercapto-3-phenyltriazole and potassium thiocyanate with 2,2-dicyanooxiranes
}

\author{
Mohammad Seifi and Hassan Sheibani* \\ Department of Chemistry, Shahid Bahonar University of Kerman, Kerman 76169, Iran \\ E-mail: $\underline{\text { hsheibani@mail.uk.ac.ir }}$
}

\begin{abstract}
A group of heterocyclic compounds of condensed [1,3]thiazole with benzimidazole or triazole derivatives were obtained by the regioselective reactions of the 2,2-dicyanooxiranes with $1 H$ 1,3-benzimidazol-2-thiol or 5-phenyl-4H-1,2,4-triazol-3-thiol respectively in good to excellent yields. Also, we performed a reaction between 2,2-oxiranedicarbonitrile reagents and potassium thiocyanate in acetic anhydride at room temperature and isolated 2-acetylimino-1,3-oxathiole derivatives.
\end{abstract}

Keywords: 2,2-Dicyanooxiranes, $1 H$-1,3-benzimidazol-2-thiols, 5-phenyl-4H-1,2,4-triazol-3thiols, potassium thiocyanate

\section{Introduction}

The design, synthesis and property of novel thiazole derivatives as a class of the most important heterocyclic compounds have been paid extensive and continuous attention in the fields of industry, agriculture and pharmacy. ${ }^{1,2}$ Many synthetic small molecules from different groups of heterocycles with influence on carcinogenesis have been reported and several of them are currently in clinical trials. ${ }^{3}$

Thiazolone nuclei fused to heterocyclic systems represent a very important class of compounds used mainly as intermediates for the preparation of bioactive compounds. For example, 2-arylidene-[1,3]thiazolo[3,2-a]benzimidazol-3(2H)-ones were found antibacterial agents, ${ }^{4}$ inhibitors of ubiquitin ligase ${ }^{5}$ and other enzymes, and they may be used for the treatment of viral and inflammatory diseases, neurological disorders and cancer. In addition, some isosteric thiazolo[3,4-a]benzimidazoles inhibit enteroviruses ${ }^{6}$ and tumors. ${ }^{7}$ The synthesis of such compounds has been the subject of several reviews which demonstrate the high importance of this class of compounds. ${ }^{8-13}$ Considering the importance of these compounds, many methods for the synthesis of these derivatives have been reported successively. The conventional synthesis 
involves the nucleophilic addition of 1,3-dinucleophies with 2,2-dicyanooxirane, alkyl $\alpha$ haloacetate or $\alpha$-haloketone derivatives. ${ }^{14}$ 2,2-Dicyanooxiranes and their $\alpha$-haloketone derivatives are known as very effective bielectrophile reagents in the synthesis of a large variety of heterocyclic compounds of pharmaceutical interest such as thiazoles, dithioles, imidazoles, ${ }^{17}$ 1,3-oxathioles ${ }^{18}$ and condensed imidazolo and thiazolo derivatives, ${ }^{19}$ because of their multifunctional structure. In general, nucleophilic reagents can react with these compounds regioselectively: they can attack the oxirane ring, cyano functional group to give either ring opened products, new functionalised oxiranes or different heterocycles. In our continuing interest in the synthesis of heterocycles containing nitrogen and sulfur atoms, ${ }^{20-22}$ we decided to investigate the condensation reaction of 1,3-dinucleophies with 2,2-dicyanooxiranes, which is extended to the formation of 1,3-thiazolone derivatives.

So in this paper, we describe an investigation of the condensation of potassium thiocyanate with 2,2-dicyanooxiranes which released to 1,3-oxathiole derivatives. These compounds are establish in a variety of useful heterocyclic derivatives applied as biologically active compounds and pharmaceutical products. ${ }^{23,24}$

\section{Results and Discussion}

To study the regioselectivity of 1,3-dinucleophiles such as $1 \mathrm{H}$-1,3-benzimidazol-2-thiol and 5phenyl-4H-1,2,4-triazol-3-thiol with 2,2-dicyanooxiranes, we have investigated the nucleophilic reaction of 1H-1,3-benzimidazol-2-thiol 2 with 2,2-dicyanooxiranes 1a-d to prepare of 2arylidene [1,3]thiazolo[3,2,a]benzimidazol-3(2H)-one (3a-d) derivatives in mild reaction conditions in good to excellent yields (Scheme 1).<smiles>[R]c1cc([R])c(C2OC2(C)C#N)cc1[R]</smiles>

1a-d<smiles>Sc1nc2ccccc2[nH]1</smiles>

2<smiles>[R]c1cc([R])c(C2Sc3nc4ccccc4n3C2=O)cc1[R]</smiles>

1a, 3a: $R^{1}=R^{2}=R^{3}=H$ 1b, 3b: $\mathrm{R}^{1}=\mathrm{R}^{3}=\mathrm{H}, \mathrm{R}^{2}=\mathrm{Cl}$ 1c, 3c: $\mathrm{R}^{1}=\mathrm{R}^{3}=\mathrm{H}, \mathrm{R}^{2}=\mathrm{NO}_{2}$ 1d, 3d: $\mathrm{R}^{1}=\mathrm{R}^{3}=\mathrm{OCH}_{3}, \mathrm{R}^{2}=\mathrm{H}$

\section{Scheme 1}


Encouraged by these results, we have extended these reactions by nucleophilic addition of 5substituted 5-phenyl-4H-1,2,4-triazol-3-thiol 4 on 2,2-dicyanooxiranes $\mathbf{1 b}$ and $\mathbf{1 d}$ to synthesis of 5-substituted thiazolo[3,2-b][1,2,4]triazol-6-ones 5a-b in good yields (Scheme 2).

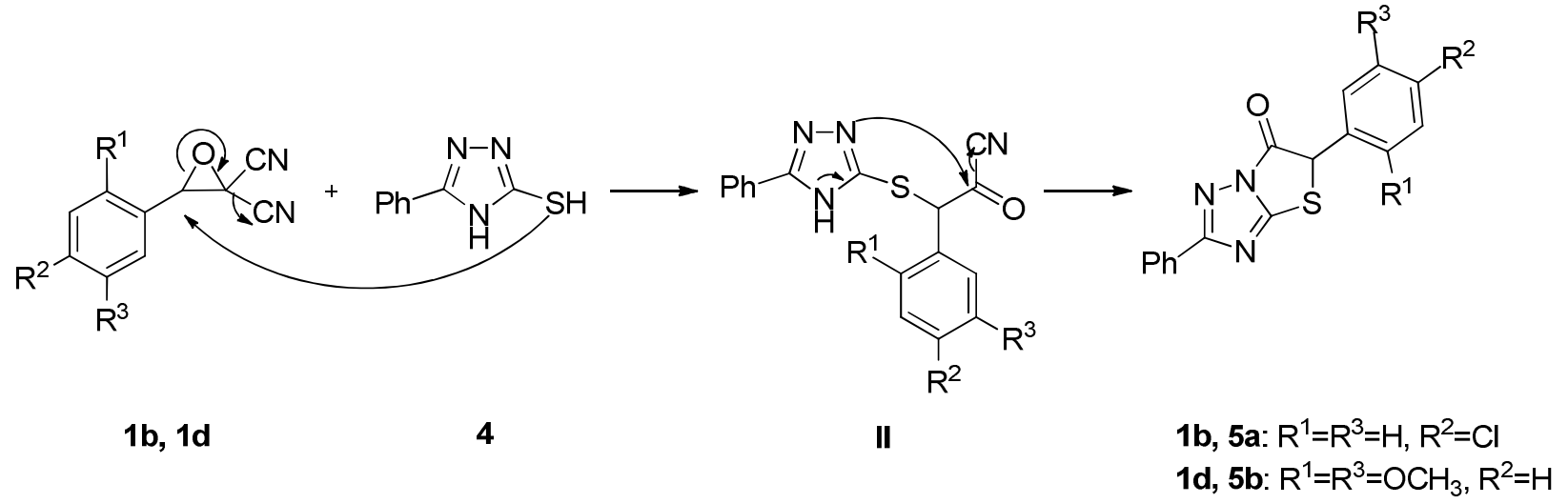

\section{Scheme 2}

In these protocols (Scheme 1 and 2) we have investigated reactions of 2,2-dicyanooxiranes $\mathbf{1}$, which have three electron-deficient centers, with 1,3-dinucleophile such as $1 H$-1,3benzimidazol-2-thiol $\mathbf{2}$ and 5-phenyl-4H-1,2,4-triazol-3-thiol 4 which afforded 2arylidene[1,3]thiazolo[3,2,a]benzimidazol-3(2H)-one 3a-d and 5-substituted thiazolo[3,2$b][1,2,4]$ triazol-6-one 5a-b derivatives, respectively. In these reactions 1,3-dinucleophiles act via regioselective on $\mathrm{C}_{\beta}$ and $\mathrm{C}_{\alpha}$ of 2,2-dicyanooxiranes $\mathbf{1}$ which have three electron deficient centers. These processes are highly regioselective, and compounds $\mathbf{3}$ and $\mathbf{5}$ results from the initial attack of the nucleophilic sulfur atom of compounds $\mathbf{2}$ and $\mathbf{4}$ on $\mathrm{C}_{\beta}$ of 2,2-dicyanooxiranes $\mathbf{1}$ followed by ring-opening to form cyanhydrin intermediate and then cyanoformyl intermediate I , II was formed by losing of cyano group. The last step is along with intramolecular nucleophilic addition of nitrogen on carbonyl group of cyanoformyl intermediate I, II and followed by the elimination of HCN to form only one product (Scheme 3).

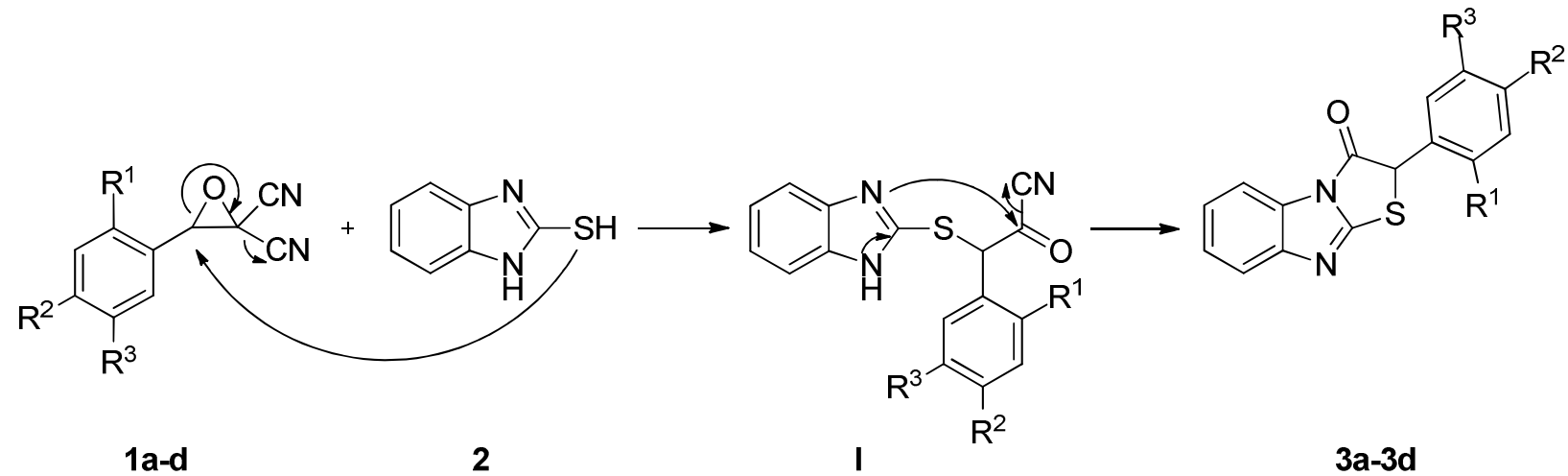

Scheme 3 
Encouraged by these results, we performed the reaction of 2,2-dicyanooxiranes (1a, $\mathbf{1 b}$ and 1d) with potassium thiocyanate 6 in acetic anhydride 7 at room temperature and isolated 2acetylimino-1,3-oxathiole derivatives 8a-c. The reaction presumably proceeds through thiocyanate attack on $\mathrm{C}_{\beta}$ of 2,2-dicyanooxiranes $\mathbf{1}$ followed by ring-opening to form cyanhydrin intermediate A. The intramolecular nucleophilic addition of oxygen on carbon of nitrile intermediate $\mathbf{A}$ leads to the formation of intermediate $\mathbf{B}$ which is trapped by acetic anhydride to yield intermediate $\mathbf{C}$. The last step is along with elimination of $\mathrm{HCN}$ from intermediate $(\mathbf{C})$ and then formation of 1,3-oxathioles 8a-c (Scheme 4).

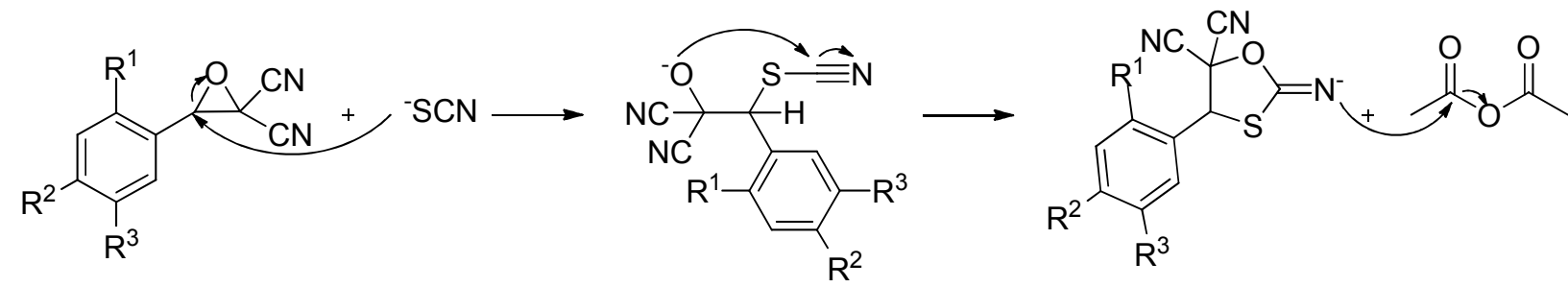

$1 a, 1 b, 1 d$

6

A
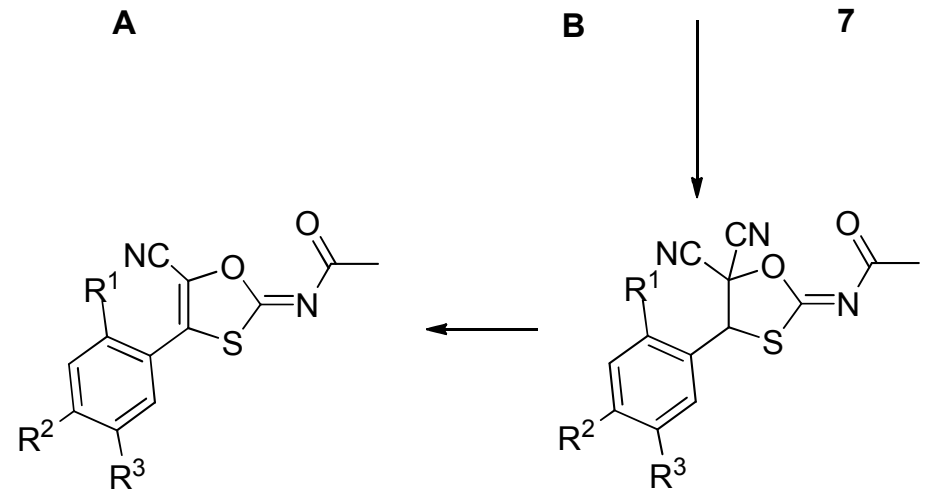

8a: $R^{1}=R^{2}=R^{3}=H$
8b: $R^{1}=R^{3}=H, R^{2}=C l$
8c: $R^{1}=R^{3}=\mathrm{OCH}_{3}, R^{2}=H$

C

\section{Scheme 4}

The structure of compounds 3a-d, 5a, 5b and 8a-c were determined on the basis of their elemental analyses, mass spectrum, ${ }^{1} \mathrm{H}$ and ${ }^{13} \mathrm{C}$ NMR and IR spectroscopic data. Only one product was obtained in each case. The ${ }^{1} \mathrm{H}$ NMR spectrum of $\mathbf{3 a}$ indicated two kinds of protons along with one signal at $6.40 \mathrm{ppm}$ attributed to one proton of $\mathrm{C}_{2}$. The aryl protons appeared at $\delta$ 8.82-7.39 ppm $(9 \mathrm{H}, \mathrm{m})$. The ${ }^{1} \mathrm{H}$ decoupled ${ }^{13} \mathrm{C}$ NMR spectrum of 3a was measured in DMSO- $d_{6}$ and it is in accord with the proposed structure. The ${ }^{13} \mathrm{C}$ NMR of compound 3a showed two signals at $\delta 153.84$ and $144.75 \mathrm{ppm}$ which are due to the carbonyl and imine carbons respectively. The ten aromatic carbons appeared at $\delta 142.92-117.76 \mathrm{ppm}$. These signals along with an upfield shift of aliphatic carbon at $\delta 50.10 \mathrm{ppm}$ which was identified as aliphatic carbon $\left(\mathrm{C}_{2}\right)$. Also the structure of compound 8a was established on the basis of spectral deta, which IR 
spectrum of this compound showed the presence of $\mathrm{CN}$ at region $2225 \mathrm{~cm}^{-1}$ and sharp bands at 1662 and $1601 \mathrm{~cm}^{-1}$, which are due to carbonyl of amide $(\mathrm{C}=\mathrm{O})$ and imine $(\mathrm{C}=\mathrm{N})$ groups respectively. The ${ }^{1} \mathrm{H}$ NMR spectrum of $\mathbf{8 a}$ indicated two kinds of proton signals with one at $\delta$ $2.66\left(\mathrm{~s}, 3 \mathrm{H}, \mathrm{CH}_{3}\right) \mathrm{ppm}$ was identified as acetyl group. The five protons of phenyl group appeared at $\delta$ 7.77-7.64 ppm. The ${ }^{13} \mathrm{C}$ NMR of compound 8a showed two signals at $\delta 181.95$ and 174.06 ppm which are due to the carbonyl of amide $(\mathrm{C}=\mathrm{O})$ and imine $(\mathrm{C}=\mathrm{N})$ groups respectively. The oxegen and cyano groups adjacent to double bond strongly polarize the double bond system. The $\alpha$-carbon $\left(\mathrm{C}_{5}\right)$ and $\beta$-carbon $\left(\mathrm{C}_{4}\right)$ of these substituents appear at $\delta 138.41$ and $114.67 \mathrm{ppm}$ respectively. The aromatic carbons along with nitryl carbon appeared at $\delta 131.79,129.82$, $127.64,126.34$ and $110.39(\mathrm{CN})$ ppm respectively. These signals along with the upfield shift of aliphatic carbon at $\delta 27.08 \mathrm{ppm}$ which was identified as acetyl group.

\section{Conclusions}

We have developed a simple and efficient method for the synthesis of heterocyclic compounds which condensed [1,3]thiazole with benzimidazole or triazole derivatives via reaction of 2,2dicyanooxiranes with 1,3-dinucleophiles. We have investigated reaction of potassium thiocyanate with 2,2-dicyanooxiranes in acetic anhydride to synthesis of oxathiole derivatives. The advantage of these procedures reported here are: high selectivity, high purity of products and easy workup.

\section{Experimental Section}

General. Melting points were measured on a Electrothermal Enginering LTD apparatus and are uncorrected. IR spectra were measured on a Mattson 1000 FT-IR spectrometer. The proton and carbon NMR spectra were recorded with a BRUKER DRX-400 AVANCE spectrometer at 400 and $100 \mathrm{MHz}$, respectively. Mass spectra were recorded on a MS-QP2000A Shimadzu mass spectrometer operating at an ionization potential of $70 \mathrm{eV}$. Elemental analyses were performed using a Heracus CHN-O-Rapid analyzer. 2,2-dicyanooxiranes 1a-d was prepared according to a literature procedure. ${ }^{25}$

Procedure for the preparation of 2 -arylidene $[1,3]$ thiazolo[3,2-a]benzimidazol-3(2H)-one (3a-d) and 5-substituted thiazolo[3,2-b][1,2,4]triazol-6-ones (5a-b). A mixture of 2,2dicyanooxiranes 1a-d $(2 \mathrm{mmol})$ and $1 H$-benzo[d]imidazole-2-thiol 2 or 5-mercapto-3-phenyl-striazole 4 ( $2 \mathrm{mmol})$ in $\mathrm{CH}_{3} \mathrm{CN}(20 \mathrm{~mL})$ was stirred for 7 hour (the progress of the reaction being monitored by TLC and was used hexane/ethyl acetate as an eluent). When the reaction was completed as indicated by TLC, the crude product $\mathbf{3 a - 3 d}$ and $\mathbf{5 a}, \mathbf{5 b}$ was precipitated from the reaction mixture, and the solid was filtered and recrystallized with hexane/ethyl acetate to get pure product. 
2-Phenyl[1,3]thiazolo[3,2-a]benzimidazol-3(2H)-one (3a). White crystals; yield: $90 \%$. $\mathrm{mp}$ 190-192 ${ }^{\circ} \mathrm{C}$. IR $\left(\mathrm{KBr}, v_{\max } / \mathrm{cm}^{-1}\right): 1742(\mathrm{C}=\mathrm{O}), 1603(\mathrm{C}=\mathrm{N}) .{ }^{1} \mathrm{H}$ NMR $\left(400 \mathrm{MHz}\right.$, DMSO- $\left.d_{6}\right)$ : $8.81\left(\mathrm{~d}, 1 \mathrm{H},{ }^{3} J_{\mathrm{H}-\mathrm{H}}=8 \mathrm{~Hz}, \mathrm{CH}\right), 7.71\left(\mathrm{~d}, 1 \mathrm{H},{ }^{3} J_{\mathrm{H}-\mathrm{H}}=8 \mathrm{~Hz}, \mathrm{CH}\right), 7.67-7.39(\mathrm{~m}, 7 \mathrm{H}, \mathrm{Ar}), 6.40(\mathrm{~s}$, 1H). ${ }^{13} \mathrm{C}$ NMR (100 MHz, DMSO- $\left.d_{6}\right): 153.84(\mathrm{C}=\mathrm{O}), 144.75(\mathrm{C}=\mathrm{N}), 142.92,133.21,132.69$, 132.38, 129.45, 129.04, 128.93, 124.63, 122.87, 117.76, $50.10\left(\mathrm{C}_{2}\right) . \mathrm{MS}(\mathrm{m} / \mathrm{z}): 266\left(\mathrm{M}^{+}\right)(98)$, 237 (80), 205 (20), 150 (15), 121 (100), 90 (78), 77 (40). Anal. calcd. for $\mathrm{C}_{15} \mathrm{H}_{10} \mathrm{~N}_{2} \mathrm{OS}$ : C, 67.65; H, 3.78; N, 10.52\%. Found: C, 67.42; H, 3.66; N, 10.18\%.

2-(4-Cholorophenyl)[1,3]thiazolo[3,2-a]benzimidazol-3(2H)-one (3b). White crystals; yield: 93\%. mp 136-139 ${ }^{\circ} \mathrm{C}$. IR $\left(\mathrm{KBr}, v_{\max } / \mathrm{cm}^{-1}\right)$ : $1726(\mathrm{C}=\mathrm{O}), 1609(\mathrm{C}=\mathrm{N}) .{ }^{1} \mathrm{H}$ NMR $(400 \mathrm{MHz}$, DMSO- $\left.d_{6}\right): 7.87\left(\mathrm{~d}, 1 \mathrm{H},{ }^{3} J_{\mathrm{H}-\mathrm{H}}=8 \mathrm{~Hz}, \mathrm{CH}\right), 7.66\left(\mathrm{~d}, 1 \mathrm{H},{ }^{3} J_{\mathrm{H}-\mathrm{H}}=8 \mathrm{~Hz}, \mathrm{CH}\right), 7.62\left(\mathrm{~d}, 2 \mathrm{H},{ }^{3} J_{\mathrm{H}-\mathrm{H}}=\right.$ $8.8 \mathrm{~Hz}, \mathrm{CH}), 7.50\left(\mathrm{~d}, 2 \mathrm{H},{ }^{3} J_{\mathrm{H}-\mathrm{H}}=8.8 \mathrm{~Hz}, \mathrm{CH}\right), 7.42\left(\mathrm{t}, 1 \mathrm{H},{ }^{3} J_{\mathrm{H}-\mathrm{H}}=8 \mathrm{~Hz}, \mathrm{CH}\right), 7.35\left(\mathrm{~d}, 1 \mathrm{H},{ }^{3} J_{\mathrm{H}-\mathrm{H}}\right.$ $=8 \mathrm{~Hz}, \mathrm{CH}), 6.32(\mathrm{~s}, 1 \mathrm{H}) \cdot{ }^{13} \mathrm{C}$ NMR $\left(100 \mathrm{MHz}, \mathrm{DMSO}-d_{6}\right): 166.83(\mathrm{C}=\mathrm{O}), 156.88(\mathrm{C}=\mathrm{N})$, 149.42, 133.61, 133.44, 131.17, 128.72, 128.02, 125.77, 123.74, 123.35, 118.70, $67.32\left(\mathrm{C}_{2}\right)$. MS $(\mathrm{m} / \mathrm{z}): 300\left(\mathrm{M}^{+}\right)$(100), $271(70), 237(50), 156(80), 124(20), 89$ (73), 63 (40). Anal. calcd. for $\mathrm{C}_{15} \mathrm{H}_{9} \mathrm{ClN}_{2} \mathrm{OS}: \mathrm{C}, 59.90 ; \mathrm{H}, 3.02 ; \mathrm{N}, 9.31 \%$. Found: C, 59.83; H, 2.88; N, 9.01\%.

2-(4-Nitrophenyl)[1,3]thiazolo[3,2-a]benzimidazol-3(2H)-one (3c). White crystals; yield: 94\%. mp 193-196 ${ }^{\circ} \mathrm{C}$. IR $\left(\mathrm{KBr}, v_{\max } / \mathrm{cm}^{-1}\right)$ : $1750(\mathrm{C}=\mathrm{O}), 1609(\mathrm{C}=\mathrm{N}) .{ }^{1} \mathrm{H}$ NMR $(400 \mathrm{MHz}$, DMSO- $\left.d_{6}\right): 8.49\left(\mathrm{~d}, 2 \mathrm{H},{ }^{3} J_{\mathrm{H}-\mathrm{H}}=8 \mathrm{~Hz}, \mathrm{CH}\right), 7.72\left(\mathrm{~d}, 1 \mathrm{H},{ }^{3} J_{\mathrm{H}-\mathrm{H}}=8 \mathrm{~Hz}, \mathrm{CH}\right), 7.50-7.26(\mathrm{~m}, 5 \mathrm{H}$, Ar), $5.79(\mathrm{~s}, 1 \mathrm{H}) .{ }^{13} \mathrm{C}$ NMR (100 MHz, DMSO- $\left.d_{6}\right): 165.83(\mathrm{C}=\mathrm{O}), 153.20(\mathrm{C}=\mathrm{N}), 150.3,145.4$, $140.01,138.5,129.73,126.56,124.55,124.49,119.51,128.9,57.61\left(\mathrm{C}_{2}\right) . \mathrm{MS}(\mathrm{m} / \mathrm{z}): 311\left(\mathrm{M}^{+}\right)$ (100), 282 (20), 265 (15), 236 (40), 167 (98), 151 (20), 145 (50), 121 (30), 109 (20), 90 (77), 77 (80), 63 (88). Anal. calcd. for $\mathrm{C}_{15} \mathrm{H}_{9} \mathrm{~N}_{3} \mathrm{O}_{3} \mathrm{~S}: \mathrm{C}, 57.87 ; \mathrm{H}, 2.91 ; \mathrm{N}, 13.50 \%$. Found: C, 57.69; H, $2.85 ; \mathrm{N}, 13.21 \%$.

2-(2,5-Dimethoxyphenyl)[1,3]thiazolo[3,2-a]benzimidazol-3(2H)-one (3d). Yellow crystals; yield: 90\%. mp 140-143 ${ }^{\circ} \mathrm{C}$. IR $\left(\mathrm{KBr}, v_{\max } / \mathrm{cm}^{-1}\right): 1748(\mathrm{C}=\mathrm{O}), 1611(\mathrm{C}=\mathrm{N}) .{ }^{1} \mathrm{H}$ NMR $(400 \mathrm{MHz}$, DMSO- $\left.d_{6}\right): 7.81\left(\mathrm{~d}, 1 \mathrm{H},{ }^{3} J_{\mathrm{H}-\mathrm{H}}=8 \mathrm{~Hz}, \mathrm{CH}\right), 7.49\left(\mathrm{~d}, 1 \mathrm{H},{ }^{3} J_{\mathrm{H}-\mathrm{H}}=8 \mathrm{~Hz}, \mathrm{CH}\right), 7.31-7.12(\mathrm{~m}, 2 \mathrm{H}$, $\mathrm{CH}), 7.05(\mathrm{~s}, 1 \mathrm{H}, \mathrm{CH}), 7.02\left(\mathrm{~d}, 1 \mathrm{H},{ }^{3} J_{\mathrm{H}-\mathrm{H}}=8 \mathrm{~Hz}, \mathrm{CH}\right), 6.88\left(\mathrm{~d}, 1 \mathrm{H},{ }^{3} J_{\mathrm{H}-\mathrm{H}}=8 \mathrm{~Hz}, \mathrm{CH}\right), 6.01(\mathrm{~s}$, $1 \mathrm{H}), 3.65,3.48\left(\mathrm{~s}, 6 \mathrm{H}, 2 \mathrm{OCH}_{3}\right) .{ }^{13} \mathrm{C}$ NMR $\left(100 \mathrm{MHz}, \mathrm{DMSO}-d_{6}\right): 167.91(\mathrm{C}=\mathrm{O}), 154.70(\mathrm{C}=\mathrm{N})$, $152.59,150.56,126.40,124.75,124.38,123.38,119.61,117.79,116.30,56.88,56.09\left(2 \mathrm{OCH}_{3}\right)$ $57.61\left(\mathrm{C}_{2}\right)$. MS (m/z): $326\left(\mathrm{M}^{+}\right)$(100), $311(85), 267(20), 178(15), 163(50), 149(60), 135(20)$, 121 (40), 107 (12), 91 (30), 77 (43), 63 (35). Anal. calcd. for $\mathrm{C}_{17} \mathrm{H}_{14} \mathrm{~N}_{2} \mathrm{O}_{3} \mathrm{~S}: \mathrm{C}, 62.56 ; \mathrm{H}, 4.32 ; \mathrm{N}$, $8.58 \%$. Found: C, 62.44; $\mathrm{H}, 4.28 ; \mathrm{N}, 8.31 \%$.

5-(4-Chlorophenyl)-2-phenylthiazolo[3,2-b][1,2,4]triazol-6(5H)-one (5a). White crystals; yield: $90 \%$. mp 152-155 ${ }^{\circ} \mathrm{C}$. IR $\left(\mathrm{KBr}, v_{\max } / \mathrm{cm}^{-1}\right): 1744(\mathrm{C}=\mathrm{O}), 1692,1611(\mathrm{C}=\mathrm{N}) .{ }^{1} \mathrm{H}$ NMR $(400$ MHz, DMSO- $\left.d_{6}\right): 8.13-8.11(\mathrm{~m}, 2 \mathrm{H}, \mathrm{Ar}), 7.64\left(\mathrm{~d}, 2 \mathrm{H},{ }^{3} J_{\mathrm{H}-\mathrm{H}}=8 \mathrm{~Hz}, \mathrm{CH}\right), 7.57-7.53(\mathrm{~m}, 3 \mathrm{H}, \mathrm{Ar})$, $7.50\left(\mathrm{~d}, 2 \mathrm{H},{ }^{3} J_{\mathrm{H}-\mathrm{H}}=8 \mathrm{~Hz}, \mathrm{CH}\right), 6.41(\mathrm{~s}, 1 \mathrm{H}) .{ }^{13} \mathrm{C}$ NMR $\left(100 \mathrm{MHz}, \mathrm{DMSO}-d_{6}\right): 169.73(\mathrm{C}=\mathrm{O})$, 165.03, $163.96(\mathrm{C}=\mathrm{N}), 134.00,132.56,131.35,131.27,129.15,129.04,128.97,126.90,58.17$ $\left(\mathrm{C}_{5}\right)$. MS (m/z): $327\left(\mathrm{M}^{+}\right)(80), 301(12), 281$ (12), 264 (55), 177 (20), $152(60), 125(40), 103$ (100), 89 (85), 77 (30). Anal. calcd. for $\mathrm{C}_{16} \mathrm{H}_{10} \mathrm{ClN}_{3} \mathrm{OS}$ : C, 58.63; H, 3.07; N, 12.28\%. Found: C, $58.44 ; \mathrm{H}, 2.98 ; \mathrm{N}, 11.97 \%$. 
5-(2,5-Dimethoxyphenyl)-2-phenylthiazolo[3,2-b][1,2,4]triazol-6(5H)-one $\quad(5 \mathrm{~b})$. Orange crystals; yield: $94 \%$. mp: $204-206{ }^{\circ} \mathrm{C}$. IR $\left(\mathrm{KBr}, v_{\max } / \mathrm{cm}^{-1}\right): 1754(\mathrm{C}=\mathrm{O}), 1606(\mathrm{C}=\mathrm{N}) .{ }^{1} \mathrm{H}$ NMR (400 MHz, DMSO- $\left.d_{6}\right): 8.13\left(\mathrm{~d}, 2 \mathrm{H},{ }^{3} J_{\mathrm{H}-\mathrm{H}}=8 \mathrm{~Hz}, \mathrm{CH}\right), 7.70-7.66(\mathrm{~m}, 3 \mathrm{H}, \mathrm{Ar}), 7.20$ (s, 1H, CH), 7.05-7.00 (m, 2H, CH), $6.31(\mathrm{~s}, 1 \mathrm{H}), 3.76,3.67\left(\mathrm{~s}, 6 \mathrm{H}, 2 \mathrm{OCH}_{3}\right) .{ }^{13} \mathrm{C}$ NMR $(100 \mathrm{MHz}, \mathrm{DMSO}-$ $\left.d_{6}\right)$ : $169.09(\mathrm{C}=\mathrm{O}), 164.73,164.32(\mathrm{C}=\mathrm{N}), 153.08,151.17,131.19,129.10,129.05,126.88$, 122.58, 117.28, 115.78, 56.45, $55.84\left(2 \mathrm{OCH}_{3}\right), 55.67\left(\mathrm{C}_{5}\right) . \mathrm{MS}(\mathrm{m} / \mathrm{z}): 353\left(\mathrm{M}^{+}\right)(12), 294(7)$, 178 (30), 163 (95), 147 (20), 135 (23), 121 (15), 103 (100), 92 (18), 77 (28). Anal. calcd. for $\mathrm{C}_{18} \mathrm{H}_{15} \mathrm{~N}_{3} \mathrm{O}_{3} \mathrm{~S}$ : C, 61.18; H, 4.28; N, 11.89\%. Found: C, 60.95; H, 4.15; N, $11.60 \%$.

Procedure for preparation of 2-acetylimino-1,3-oxathiole derivatives 8a-c. A mixture of 2,2dicyanooxiranes 1a-d $(2 \mathrm{mmol})$ and $\mathrm{KSCN}(2 \mathrm{mmol})$ was dissolved in $10 \mathrm{ml}$ of acetic anhydride and stirred at room temperature for $3 \mathrm{~h}$. The product precipitated from the reaction mixture and was collected by filtration. It was recrystallized from EtOH.

(5E)-N-(5-Cyano-4-phenyl)-1,3-oxathiol-2-ylidene)acetamide (8a). Brown crystals; yield: 90\%. mp 97-99 ${ }^{\circ} \mathrm{C}$. IR (KBr, $\left.v_{\max } / \mathrm{cm}^{-1}\right): 2225(\mathrm{CN}), 1662(\mathrm{C}=\mathrm{O}), 1601(\mathrm{C}=\mathrm{N}) .{ }^{1} \mathrm{H}$ NMR $(400$ MHz, DMSO- $\left.d_{6}\right)$ : 7.77-7.74 (m, 3H, Ar), $7.64\left(\mathrm{~d}, 2 \mathrm{H},{ }^{3} \mathrm{~J}_{\mathrm{H}-\mathrm{H}}=8 \mathrm{~Hz}, \mathrm{CH}\right), 2.66\left(\mathrm{~s}, 3 \mathrm{H}, \mathrm{CH}_{3}\right) .{ }^{13} \mathrm{C}$ NMR (100 MHz, DMSO- $\left.d_{6}\right)$ : $181.95(\mathrm{C}=\mathrm{O}), 174.06(\mathrm{C}=\mathrm{N}), 138.41\left(\mathrm{C}_{5}\right), 131.79,129.82,127.64$, 126.34, $114.67\left(\mathrm{C}_{4}\right), 110.39(\mathrm{CN}), 27.08\left(\mathrm{CH}_{3}\right) . \mathrm{MS}(\mathrm{m} / \mathrm{z}): 244\left(\mathrm{M}^{+}\right)(7), 193(8), 168(15)$, 135(18), 121 (100), 107 (10), 89 (45), 77 (85). Anal. calcd. for $\mathrm{C}_{12} \mathrm{H}_{8} \mathrm{~N}_{2} \mathrm{O}_{2} \mathrm{~S}: \mathrm{C}, 59.00 ; \mathrm{H}, 3.30$; N, 11.47\%. Found: C, 58.79; H, 3.21; N, 11.12\%.

(5E)-N-(4-(4-Chlorophenyl)-5-cyano-1,3-oxathiol-2-ylidene)acetamide (8b). Yellow crystals; yield: 95\%. mp117-120 ${ }^{\circ} \mathrm{C}$. IR (KBr, $\left.v_{\max } / \mathrm{cm}^{-1}\right): 2229(\mathrm{CN}), 1666(\mathrm{C}=\mathrm{O}), 1599(\mathrm{C}=\mathrm{N}) .{ }^{1} \mathrm{H}$ NMR $\left(400 \mathrm{MHz}, \mathrm{DMSO}-d_{6}\right): 7.77\left(\mathrm{~d}, 2 \mathrm{H},{ }^{3} J_{\mathrm{H}-\mathrm{H}}=8 \mathrm{~Hz}, \mathrm{CH}\right), 7.72\left(\mathrm{~d}, 2 \mathrm{H},{ }^{3} J_{\mathrm{H}-\mathrm{H}}=8 \mathrm{~Hz}, \mathrm{CH}\right), 2.36(\mathrm{~s}$, $\left.3 \mathrm{H}, \mathrm{CH}_{3}\right) .{ }^{13} \mathrm{C}$ NMR (100 MHz, DMSO-d $): 182.66(\mathrm{C}=\mathrm{O}), 163.37(\mathrm{C}=\mathrm{N}), 149.82\left(\mathrm{C}_{5}\right), 136.97$, 136.46, 129.90, 128.63, $124.29\left(\mathrm{C}_{4}\right), 110.22(\mathrm{CN}), 26.70\left(\mathrm{CH}_{3}\right) . \mathrm{MS}(\mathrm{m} / \mathrm{z}): 278\left(\mathrm{M}^{+}\right)(90), 237$ (8), 209 (8), 180(10), 155 (100), 114 (80), 93 (40), 70 (65). Anal. calcd. for $\mathrm{C}_{12} \mathrm{H}_{7} \mathrm{ClN}_{2} \mathrm{O}_{2} \mathrm{~S}$ : C, $51.71 ; \mathrm{H}, 2.53 ; \mathrm{N}, 10.05 \%$. Found: C, 51.48; H, 2.27; N, 9.71\%.

(5E)- $N$-(5-Cyano-4-(2,5-dimethoxyphenyl)-1,3-oxathiol-2-ylidene)acetamide (8c). Yellow crystals; yield: $92 \%$. mp 186-189 ${ }^{\circ} \mathrm{C}$. IR (KBr, $\left.v_{\max } / \mathrm{cm}^{-1}\right): 2229(\mathrm{CN}), 1656(\mathrm{C}=\mathrm{O}), 1609(\mathrm{C}=\mathrm{N})$. ${ }^{1} \mathrm{H}$ NMR (400 MHz, DMSO- $\left.d_{6}\right)$ : 7.26-7.20 (m, 3H, Ar), 3.86, 3.77 (s, 6H, 20CH $), 2$ (s, 3H, $\left.\mathrm{CH}_{3}\right) .{ }^{13} \mathrm{C}$ NMR (100 MHz, DMSO-d $\left.d_{6}\right): 181.95(\mathrm{C}=\mathrm{O}), 174.36(\mathrm{C}=\mathrm{N}), 153.14\left(\mathrm{C}_{5}\right), 152.77$, $150.62,118.68,115.42,114.52,113.85,113.40\left(\mathrm{C}_{4}\right), 110.323(\mathrm{CN}), 56.34,55.70\left(2 \mathrm{OCH}_{3}\right)$, $26.89\left(\mathrm{CH}_{3}\right)$. MS (m/z): $304\left(\mathrm{M}^{+}\right)$(10), 262 (30), 235 (25), 181 (100), 149 (90), 133 (40), 121 (70), 107 (18), 95 (20), 77 (8). Anal. calcd. for $\mathrm{C}_{14} \mathrm{H}_{12} \mathrm{~N}_{2} \mathrm{O}_{4} \mathrm{~S}$ : C, 55.52; H, 3.97; N, 9.21\%. Found: C, 55.39; H, 3.89; N, 8.94\%.

\section{Acknowledgements}

The authors express appreciation to the Shahid Bahonar University of Kerman Faculty Research Committee for its support of this investigation. 


\section{References}

1. Ares, J. J. Synth. Commun. 1991, 21, 625. http://dx.doi.org/10.1080/00397919108020829

2. Fang, D.; Wei, H.; Luyt, A. S. Chin. Chem. Lett. 2011, 22, 109. http://dx.doi.org/10.1016/j.cclet.2010.09.019

3. Eckhardt, S. Curr. Med. Chem. Anticancer Agents 2002, 2, 419. http://dx.doi.org/10.2174/1568011024606389 PMid:12678741

4. Allanson,N. M.; Leslie, B. W.; Thomson, S. Pat. UK. Application 2002, 12, 31.

5. Alroy, I.; Tuvia, Sh.; Reiss, Levi-Hacham, YO. Pat. USA. Application 2007, 18, 1.

6. Leyssen, P.; De Clercq, E.; Neyts, J. Antivir. Res. 2008, 78, 9. http://dx.doi.org/10.1016/j.antiviral.2008.01.004 PMid:18313769

7. Monforte, P.; Musumeci, L.; Rao, A.; Zappala, M.; Monforte, A. M. Arch. Pharm. 2001, 334, 203.

8. Franis, J. E.; Cashi, W.; Psychoyos, D. S.; Wenk,G. P.; Friedmann, R. C.; Atkins, C.; Warren, W.; Furness, P.; Hyun, J. L.; Stone, G. A.; Desai, M.; Williams, M. J. Med. Chem. 1988, 31, 1014. http://dx.doi.org/10.1021/jm00400a022

9. Bentabed-Ababsa, G.; Hamza-Reguig, S.; Derdour, A.; Domingo, L. R.; Saez, J. A.; Roisnel, T.; Dorcet, V.; Nassar, V.; Mongin, F. Org. Bio. Chem. 2012, 10, 8434. http://dx.doi.org/10.1039/c2ob26442k PMid:23011385

10. Ghailane, T.; Saadouni, M.; Boukhris, S.; Habbadi, N.; Hassikou, A.; Kerbal, A.; Garrigues, B.; Souizi, A. Heterocycles 2011, 83, 357. http://dx.doi.org/10.3987/COM-10-12096

11. Bentabed-Ababsa, G.; Derdour, A.; Roisnel, T.; Saez, J. A.; Perez P.; Chamorro E.; Domingo, L. R.; Mongin, F. J. Org. Chem. 2009, 74, 2120.

http://dx.doi.org/10.1021/jo8027104

PMid:19199802

12. Bentabed-Ababsa, G.; Derdour, A.; Roisnel, T.; Saez, J. A.; Domingo, L. R.; Mongin, F. Org. Bio. Chem. 2008, 6, 3144. http://dx.doi.org/10.1039/b804856h PMid:18698474

13. Kim, Y. C.; Ji, X. D.; Jacobson, K. A. J. Med. Chem. 1996, 39, 4142. http://dx.doi.org/10.1021/jm960482i PMid:8863790

14. Robert, A.; Baudy-Floc'h, M.; Le Grel, P.; Foucaud, A. Trends in Org. Chem. 1995, 5, 37. 
15. Majcen-Le Maréchal, A.; Robert, A.; Leban, I. Tetrahedron 1990, 46, 453.

http://dx.doi.org/10.1016/S0040-4020(01)85429-6

16. Souizi, A.; Robert,A. C. R. Acad. Sci., Paris Ser. 1982, 295, 571.

17. Guillemet, M.; Robert, A.; Baudy-Floc'h, M. Tetrahedron Lett. 1995, 36, 547.

http://dx.doi.org/10.1016/0040-4039(94)02337-B

18. Majcen-Le Maréchal, A.; Robert, A.; Leban, I. J. Chem. Soc., Perkin Trans. 1993, 1, 351.

19. Majcen-Le., Maréchal, A.; Le Grel, P.; Robert, A.; Biškup, J.; Ferk, V.; Toplak, R. Arkivoc 2001, (v), 119.

20. Cantillo, D.; Sheibani, H.; Kappe, C. O. J. Org. Chem. 2012, 77, 2463.

http://dx.doi.org/10.1021/jo3001645

PMid:22321044

21. Sheibani, H.; Hassani, F. J. Heterocyclic. Chem. 2011, 48, 915.

http://dx.doi.org/10.1002/jhet.640

22. Sheibani, H.; Amrollahi, M. A.; Esfandiarpoor, Z. Mol Divers. 2010, 14, 277.

http://dx.doi.org/10.1007/s11030-009-9165-5

PMid: 19543800

23. Konieczny, M. T.; Konieczny, W.; Sabisz, M.; Skladanowski, A.; Wakiec, R.; Augustynowicz -Kopec, E.; Zwolska, Z. Chem. Pharm. Bull. 2007, 55, 817.

24. Aizawa, Y.; Kanai, T.; Hasegawa, K.; Yamaguchi, T.; Iizuka, T.; Iwaoka, T.; Yoshioka, T. J. Med. Chem. 1990, 33, 1491.

http://dx.doi.org/10.1021/jm00167a032

25. Volmajer, J.; Toplak, R.; Bittner, S.; Marechal, A. M. L. Arkivoc 2003, (xiv), 49.

http://dx.doi.org/10.3998/ark.5550190.0004.e06 